\title{
Importancia del cuadro básico de medicamentos en la prescripción médica
}

\author{
Luis Jasso, Alberto Lifshitz, Oscar Arrieta, Rubén Burgos, Carlos Campillo, Miguel Á. Celis, \\ Manuel de la Llata, Judith Domínguez, José Halabe, Sergio Islas, Mucio Moreno, Ricardo Plancarte, \\ Alejandro Reyes-Sánchez, Guillermo Ruiz-Argüelles, Antonio Soda, Julio Sotelo* y Emma Verástegui \\ Academia Nacional de Medicina, Comité de Ética y Transparencia en la Relación Médico-Industria (CETREMI), Ciudad de México, México
}

\begin{abstract}
Resumen
La implementación en instituciones de salud de un cuadro básico permite adquirir y administrar una larga lista de medicamentos que presenta a los médicos las alternativas de tratamiento, así como la descripción académica colegiada de indicaciones, dosis, efectos secundarios, interacciones y análisis de costo-beneficio, con lo que se facilita la prescripción médica y la administración de insumos para la salud. El Comité de Ética y Transparencia en la Relación Médico-Industria emite diversas recomendaciones para la optimización de los beneficios generados por los cuadros básico de medicamentos.
\end{abstract}

PALABRAS CLAVE: Cuadro básico. Costo de medicamentos. Prescripción. Costo-beneficio. Nuevos fármacos.

\section{Importance of the list of essential medicines in medical prescription}

\begin{abstract}
The implementation of an essential medicines list in health institutions allows acquiring and administering a long list of drugs that offers treatment alternatives to physicians, as well as a collegiate academic description of indications, doses, side effects, interactions and cost-benefit analyses, thus facilitating medical prescription and administration of health products. The Committee of Ethics and Transparency in the Physician-Industry Relationship issues several recommendations for optimizing the benefits generated by essential medicines lists.
\end{abstract}

KEY WORDS: Essential medicines list. Medication cost. Prescription. Cost-benefit. New drugs.

Los cuadros básicos de medicamentos (los hay también de equipo y de otros suministros) han sido valiosos auxiliares para la administración en las instituciones de salud. Actualmente, por razones simbólicas que consideran solo sus inconvenientes, se tiende a cambiarles de nombre por uno equivalente tales como catálogo, índice o compendio, aunque su orientación principal es administrativa, pues su mayor utilidad radica en que los administradores institucionales sepan qué comprar y acotar estudios de impacto en la calidad o costos de atención. Lo cierto es que los cuadros básicos de medicamentos influyen indudablemente en las prescripciones de los médicos. Aquí se comentarán aspectos médicos, intentando definir una postura del Comité de Ética y Transparencia en la Relación Médico-Industria (CETREMI) ${ }^{1}$ de la Academia Nacional de Medicina.

La principal crítica a los cuadros básicos es que restringen la libertad de prescripción, bajo el argumento de que es una norma restrictiva manipulada
Correspondencia:

*Julio Sotelo

E-mail: jsotelo@unam.mx

DOI: 10.24875/GMM.20005677

0016-3813/๑ 2020 Academia Nacional de Medicina de México,

CC BY-NC-ND (http://creativecommons.org/licenses/by-nc-nd/4.0/).
Fecha de recepción: 11-12-2019

Fecha de aceptación: 03-01-2020
Gac Med Mex. 2020;156:610-611

Disponible en PubMed

www.gacetamedicademexico.com 
por intereses farmacéuticos y que en las instituciones de salud no pueden utilizarse medicamentos no incluidos en el citado catálogo.

De igual forma, se deben definir las ventajas del cuadro básico en la calidad del servicio.

La inclusión de los medicamentos en los cuadros básicos se basa en un consenso entre las instituciones de salud, que en buena medida previene la adquisición de medicamentos inútiles o con utilidad no comprobada, como existen muchos en el mercado. Los médicos pueden ver en el cuadro básico una guía generada por expertos que incluye los medicamentos útiles que poseen buenos índices de costo-beneficio; además contiene información sobre indicaciones, vías de administración, dosis, algoritmos clínicos, efectos adversos e interacciones. Es, en cierto modo, una guía de prescripción no sesgada por intereses comerciales. Es claro que un buen cuadro básico debe tener flexibilidad para incorporar oportunamente innovaciones y permitir las excepciones que se fundamenten en información sólida científicamente sustentada. Su principal utilidad es propiciar un buen abasto, ya que en ningún sistema es posible disponer de la enorme totalidad de medicamentos que ofrece el mercado (de regulación imperfecta).

Las instituciones guían sus compras y suministros mediante un catálogo de los fármacos más frecuentemente empleados, siempre y cuando tengan evidencia de eficacia y seguridad. En México, el Consejo de Salubridad General genera un cuadro básico de medicamentos del sector salud gubernamental, que ahora se denominará Compendio Nacional de Insumos para la Salud. Con esto se logra el agrupamiento y clasificación de los fármacos autorizados por el Consejo de Salubridad General y cuya adquisición por las instituciones de salud sea lo más uniforme posible, tanto en calidad como en costos. También se logra la uniformidad de aplicación de cada uno, con el enunciado de sus respectivos efectos colaterales, vías de administración, dosis y posibles efectos adversos. Al mismo tiempo, el catálogo permite realizar el cálculo de la cantidad de medicamentos y recursos monetarios que requerirá cada institución de salud.

Los cuadros básicos no tienen el propósito de limitar las decisiones de los médicos, sino de propiciar que las instituciones cuenten con los insumos necesarios para el óptimo ejercicio de la profesión, así como para intentar una racionalidad de la prescripción sin influencia de intereses comerciales. Un aspecto relevante para que el cuadro básico mantenga su máxima utilidad es su actualización frecuente, por expertos colegiados, con la incorporación o desincorporación de fármacos e indicaciones de acuerdo con resultados de investigación científica pertinente y reciente y la conveniencia por análisis de costo-beneficio.

\section{Conflicto de intereses}

Los autores declaran que no existe conflicto de intereses.

\section{Financiamiento}

Ninguno

\section{Responsabilidades éticas}

Protección de personas y animales. Los autores declaran que para esta investigación no se realizaron experimentos en seres humanos ni en animales.

Confidencialidad de los datos. Los autores declaran que en este artículo no aparecen datos de pacientes.

Derecho a la privacidad y consentimiento informado. Los autores declaran que en este artículo no aparecen datos de pacientes.

\section{Bibliografía}

1. Academia Nacional de Medicina. Comité de ética y transparencia de los médicos con la industria farmacéutica (CETREMI). Gac Med Mex. 2015;151:293. 